\title{
Fatores associados ao conhecimento e utilização de estratégias de prevenção do HIV entre mulheres trabalhadoras do sexo em 12 cidades brasileiras
}

\author{
Factors associated with knowledge and use of hiv prevention \\ strategies among female sex workers in 12 brazilian cities
}

Ana Francisca Kolling (https://orcid.org/0000-0002-3251-9048) ${ }^{1}$

Silvano Barbosa de Oliveira (https://orcid.org/0000-0002-1966-6115) ${ }^{2}$

Edgar Merchan-Hamann (https://orcid.org/0000-0001-6775-9466) ${ }^{3}$

${ }^{1}$ Ministério da Saúde, Departamento de DST, Aids e Hepaties Virais.

SAF Sul Trecho II, Bloco F Torre I Edíficio Premium Andar Auditório Sala 4, Zona Cívico-Administrativa. 70070-600 Brasília DF Brasil.

anakolling81@hotmail.com

2 Organização Pan-

Americana da Saúde (OPAS/

OMS). Brasília DF Brasil.

${ }^{3}$ Departamento de Saúde

Coletiva, Faculdade

de Ciências da Saúde,

Universidade de Brasília.

Brasília DF Brasil.

\begin{abstract}
This article aims to Identify factors associated with knowledge and use of combined HIV prevention strategies among female sex workers (FSW). Cross-sectional epidemiological study, using the Respondent Driven Sampling (RDS) method. Descriptive analyzes were performed after adjustments required by the RDS method. To investigate factors associated with knowledge of PEP and PrEP and use of the female condom, Poisson regression was applied, using odds ratio as an association measure. FSW not affiliated with NGOs, who did not receive informational material on prevention and / or participated in lectures in the last six months and who do not identify themselves as FSW in healthcare service facilities have less knowledge about PEP and PrEP and use the female condom less frequently. In general, FSW do not have sufficient knowledge and access to combined HIV prevention methods to take advantage of their benefits. We believe that the adoption of different models of care for FSW in partnership with NGOs can be an effective strategy for expanding knowledge and use of HIV prevention methods in Brazil.
\end{abstract}

Key words HIV, Sex work, Prevention
Resumo O objetivo deste artigo é identificar fatores associados ao conhecimento e utilização de estratégias de prevenção combinada do HIV entre mulheres trabalhadoras do sexo (MTS). Estudo epidemiológico transversal, utilizando o método Respondent Driven Sampling (RDS). Foram realizadas análises descritivas após ajustes requeridos pelo método RDS. Para investigar fatores associados ao conhecimento de PEP e PrEP e utilização do preservativo feminino foi usada a regressão de Poisson, utilizando como medida de associação o Odds Ratio. MTS sem vínculo com ONGs, que não receberam material informativo sobre prevenção elou participaram de palestras nos últimos seis meses e que não se identificam como MTS nos serviços de saúde têm menos conhecimento sobre PEP e PrEP e utilizam com menor frequência o preservativo feminino. De maneira geral, as MTS não têm conhecimento e acesso suficiente às tecnologias de prevenção combinada do HIV a ponto de usufruir de seus benefícios. Consideramos que a adoção de modelos diferenciados de cuidados voltados para MTS em parceria com ONGs pode ser uma estratégia efetiva para ampliação do conhecimento e utilização das tecnologias de prevenção do HIV no Brasil.

Palavras-chave HIV, Trabalho sexual, Prevenção 


\section{Introdução}

No Brasil, aproximadamente 900 mil pessoas vivem com HIV (PVHIV) e todos os anos são registrados em média 40 mil casos novos ${ }^{1,2}$. A epidemia de HIV no Brasil é considerada concentrada em alguns segmentos populacionais, incluindo as mulheres trabalhadoras do sexo (MTS) que acumulam uma taxa de prevalência estimada de 5,3\% (IC 95\%: 4,4\% -6,2\%), maior em relação à população geral $(0,4 \%)^{1,3}$.

Outro dado importante é o aumento da prevalência de sífilis nessa população, estimada em 2016 (8,5\%; IC95\%: 7,3\% -9,7\%) 3,5 vezes superior a 2009 (2,4\%; IC95\%: $1,7 \%$ a 3,4\%). Além disso, as estimativas de prevalência de HBV e HCV foram de 0,4\% (IC 95\%: 0,2\%-0,7\%) e 0,9\% (95\%IC: $0,6 \%-1,3 \%)^{3}$.

Consta-se no Sistema de Informações de Agravos de Notificação (SINAN), no período de 2007 a junho de 2019, um total de 207.207 (69,0\%) casos de infecção pelo HIV em homens e $93.220(31,0 \%)$ casos em mulheres. A razão de sexos para o ano de 2018 foi de 2,6 (M:F), ou seja, 26 homens para cada dez mulheres. Entre as mulheres, $37,2 \%$ dos casos se deram entre brancas e $53,6 \%$ entre negras (pretas, $12,9 \%$ e pardas, $40,7 \%)^{1}$.

Segundo Boletim Epidemiológico de HIV publicado pelo Ministério da Saúde em 2019, observou-se uma tendência de queda da taxa de detecção entre as mulheres nos últimos dez anos, que passou de 17,0 casos/100.000 habitantes em 2008, para 10,5 em 2018, representando uma redução de $38,2 \%{ }^{1}$.

Entre as mulheres, verifica-se que, nos últimos dez anos, a taxa de detecção de HIV apresentou queda em todas as faixas etárias, sendo as faixas de 25 a 29 e de 30 a 34 anos as que apresentaram as maiores quedas: $51,2 \%$ e $53,2 \%$, respectivamente, quando comparados os anos de 2008 e $2018^{1}$.

Em 2018, as faixas etárias com maior detecção foram as das mulheres entre 40 e 44 anos (20,5 casos/100.000 habitantes) ${ }^{1}$.

Na América Latina a epidemia de HIV é concentrada, estando em níveis considerados estáveis na população geral $(0,2-0,7 \%)$. No Caribe, a epidemia é generalizada e uma das mais altas taxas de prevalências de HIV do mundo $(<0,1-$ $3 \%)$, sendo que as mulheres são responsáveis pela metade de todas as infecções. O cenário epidemiológico do HIV nos países latino-americanos é de maior prevalência entre homens que fazem sexo com homens (HSH), cerca de $11 \%$ a $17 \%$ na maioria dos países, com exceção de Bolívia (25\%), México e Paraguai (21\%) que demonstram prevalências acima de $20 \%{ }^{4,5}$.

Foram registradas cem mil novas infecções de HIV na América Latina em 2017. O Brasil é responsável por 35\% do número total de PVHIV da região e por $47 \%$ das novas infecções que ocorreram nesse período. Cerca de $1 \%$ das trabalhadoras do sexo do Chile, Colômbia, Costa Rica, Guatemala, Paraguai, Peru e Uruguai viviam com HIV em 2017 em comparação com cerca de 5\% na Bolívia, Brasil e Panamá. No Caribe, a prevalência varia entre $2 \%$ na Jamaica e $6 \%$ na Guiana. Quase $90 \%$ das novas infecções nesse período ocorreram em Cuba, na República Dominicana, no Haiti e na Jamaica. O Haiti responde por quase metade das novas infecções anuais por HIV e mortes relacionadas à Aids 4 .

Estimativas mundiais demonstram que mulheres trabalhadoras do sexo podem chegar a ter 13,5 vezes mais chances de ter HIV do que outras mulheres (IC95\% 10; 0-18·1).

Estigma e discriminação são importantes barreiras que dificultam o acesso e o uso dos serviços de saúde, principalmente devido ao medo de se identificar como trabalhadora do sexo e receber um tratamento inadequado dos profissionais de saúde ${ }^{6,7}$. Estudo realizado pela RedTraSex (Red de Mujeres Trabajadoras Sexuales de Latinoamérica y el Caribe), em 14 países da América Latina, demonstra que quase dois terços das trabalhadoras do sexo entrevistadas não haviam mencionado seu trabalho na última visita ao serviço de saúde (REDTRASEX, 2013).

O trabalho sexual não é considerado crime no Brasil; a ilicitude é caracterizada quando há o envolvimento de menores de idade ou exploração sexual. Mesmo assim, essa ocupação ainda não está livre de discriminação e violações de direitos humanos ${ }^{6-8}$. O modelo abolicionista, adotado na Argentina e também no Brasil desde 1942, quando entrou em vigor o atual Código Penal, considera certo grau de omissão do Estado no que diz respeito às garantias e direitos fundamentais de quem exerce o trabalho sexual, ou seja, nesse modelo, mesmo que não haja criminalização do trabalho sexual, quem o exerce fica à margem da sociedadeno que diz respeito aos direitos trabalhistas e previdenciários ${ }^{9,10}$.

Diferente do Brasil, alguns países sul-americanos como Uruguai, Equador e Bolívia adotam o regulamentarismo como sistema jurídico. Nesse modelo a profissão é reconhecida e regulamentada, porém existem algumas exigências a serem cumpridas, como por exemplo, que a mulher se 
submeta a exames periódicos ou que só exerça a atividade em locais determinados. Algumas garantias legais também são possibilitadas como a elaboração de contratos de trabalho, seguro social e aposentadoria ${ }^{10}$.

No Brasil, as estratégias de prevenção do HIV apresentam estágios diferentes de implantação. Dados divulgados pelo Ministério da Saúde sobre a Pesquisa de Conhecimentos Atitudes e Práticas na População Brasileira (PCAP) realizada em 2013 mostram que a maioria dos brasileiros (94\%) sabe que o preservativo é melhor forma de prevenção às IST. Mesmo assim, 45\% da população sexualmente ativa do país não usou preservativo nas relações sexuais casuais nos últimos 12 meses $^{11}$.

Nos anos 1990 vivenciamos a chamada tendência de feminização da epidemia de HIV, devido à evidência do crescimento de casos entre mulheres. Para responder a este fenômeno, o preservativo feminino começou a ser distribuído pelo Sistema Único de Saúde (SUS) nos anos 2000 (NEPO, ABIA e UNFPA, 2011). A importância do preservativo feminino está relacionada principalmente ao fato de ser um insumo de prevenção sob o controle da mulher, permitindo maior autonomia em relação ao seu corpo e à sua vida sexual, especialmente em situações que envolvem negociação do uso do preservativo masculino $^{12}$.

Além das intervenções biomédicas consideradas clássicas, que incluem entre outras estratégias o uso de métodos de barreira como, por exemplo, o uso de preservativos, existe outro grupo de intervenções que estão relacionados à utilização de antirretrovirais (ARV) com o objetivo de limitar a capacidade do HIV de infectar indivíduo ${ }^{13,14}$.

A Profilaxia Pós Exposição (PEP) é a utilização de antirretrovirais (ARV) por 28 dias, após qualquer situação em que exista o risco de contato com o vírus HIV. A recomendação é de início da PEP em até 72 horas, sendo mais eficaz se iniciado nas duas primeiras horas após a exposição. A PEP está disponível no SUS para profissionais de saúde, que tiveram exposição ao vírus do HIV decorrente de acidente ocupacional com material perfurocortante, desde 1999, e sua oferta vem sendo ampliada também para outros tipos de exposição, como no caso de exposição sexual consentida e nos casos de violência sexual. Nos últimos anos a oferta de PEP aumentou de 15.540 em 2009 para 107.340 profilaxias utilizadas em $2018^{15,16}$.

O Brasil foi o primeiro país da América Latina a instituir o tratamento para todas as PVHIV, independentemente do valor do $\mathrm{CD} 4+$, desde 2013. Até 2017, 45\% dos países latino-americanos haviam adotado a mesma política de saúde, porém a cobertura de tratamento varia enormemente entre os países: de $36 \%$ na Bolívia a $67 \%$ no Peru (na América Latina) e de 31\% em Belize, para $66 \%$ em Cuba (no Caribe) ${ }^{4}$. As condições relacionadas à Aids continuam sendo as principais causas de morte entre PVHIV na região das Américas ${ }^{4,5}$.

A profilaxia pré-exposição ao HIV (PrEP) consiste na utilização de antirretrovirais por pessoas que não estão infectadas pelo HIV, mas que possam estar vivendo uma situação de vulnerabilidade ao vírus. A PrEP foi a intervenção biomédica mais recentemente incorporada ao âmbito da Prevenção Combinada no Brasil, em 2017 ${ }^{17,18}$.

A oferta de PrEP na América Latina é escassa em toda a região. O Brasil é o único país da região em que a PrEP está disponível no SUS. Mesmo em países latino-americanos que têm sistemas públicos universais de saúde não existe oferta de PrEP de forma gratuita. No Chile, Costa Rica, Guatemala, México e Uruguai, a PrEP pode ser obtida por meio de provedores privados de saúde, ou projetos de pesquisa. Bahamas e Barbados eram os únicos países do Caribe que ofertavam PrEP através de um sistema público de saúde em 2018, embora também esteja disponível no sistema privado de saúde na Jamaica e no Suriname. A PrEP ainda não está disponível em Cuba, na República Dominica e Haiti ${ }^{4}$.

Desde 2013 o Brasil vem investindo em modelos de prevenção combinada para tentar conter o avanço dos casos de HIV. Em um cenário epidemiológico de maior prevalência de HIV em alguns segmentos populacionais é importante que estes grupos possam ter acesso facilitado aos insumos de prevenção. Atualmente, o Brasil dispõe dos seguintes insumos/estratégias de prevenção combinada: oferta de preservativos femininos e masculinos, gel lubrificante, testagem regular para HIV, hepatites B e C e Sífilis; a prevenção da transmissão vertical (quando o vírus é transmitido para o bebê durante a gravidez), apoiada principalmente por ações que visam a qualificação do pré-natal; o diagnóstico e tratamento das IST e hepatites virais; a imunização para hepatites A e B; programas de redução de danos para pessoas que usam álcool e outras substâncias; a profilaxia pré exposição (PrEP); profilaxia pós exposição (PEP); e o tratamento para pessoas vivendo com HIV (PVHIV) ${ }^{13,14}$.

Dada a importância que a epidemia Brasileira de HIV tem na região das Américas, este artigo 
tem por objetivo identificar os principais fatores associados ao conhecimento e a utilização de insumos de prevenção do HIV entre trabalhadoras do sexo, fornecendo evidências que apoiem a adoção de intervenções que facilitem o acesso das MTS às estratégias de prevenção combinada.

\section{Materiais e métodos}

Trata-se de um estudo epidemiológico transversal, utilizando amostragem baseada na metodologia Respondent Driven Sampling (RDS). Esse recorte é derivado de dados coletados do estudo realizado em 2016, denominado "Projeto Corrente da Saúde II", realizado entre os meses de junho e novembro de 2016, com o objetivo de estimar aprevalência de HIV, sífilis e hepatite B e C e de avaliar conhecimentos, atitudes e práticas relacionadas à infecção pelo HIV e outras infecções sexualmente transmissíveis (IST) entre MTS 3,19,20.

As cidades e o tamanho mínimo da amostra foram estabelecidos pelo Departamento de Doenças de Condições Crônicas e Infecções Sexualmente Transmissiveis (DCCI) doMinistério da Saúde (MS), de acordo com critérios geográficos e epidemiológicos. O tamanho de amostra pré -estabelecido foi de no mínimo 350 entrevistas válidas por cidade, em alguns locais esse número foi um pouco maior totalizando 4.328 MTS entrevistadas $^{19,20}$.

Foram analisados dados de 4.328 questionários preenchidos por mulheres trabalhadoras do sexo das cidades de Manaus, Belém, Fortaleza, Recife, Salvador, Belo Horizonte, Rio de Janeiro, São Paulo, Curitiba, Porto Alegre, Campo Grande e Brasília. Os critérios de elegibilidade incluíram ser mulher biologicamente; mínimo 18 anos de idade; ser trabalhadora do sexo em uma das cidades do estudo; ter trocado sexo por dinheiro pelo menos uma vez nos últimos quatro meses anteriores a entrevista; apresentar um cupom válido; não ter participado da pesquisa anteriormente; não apresentar indício de uso de drogas ou álcool no momento da participação; aceitar o convite e assinar o Termo de Consentimento Livre e Esclarecido (TCLE). A descrição completa da metodologia utilizada foi publicada por Damacena $(2019)^{20}$.

Neste estudo utilizamos análise descritiva para conhecer as principais características relacionadas ao acesso e utilização de insumos de prevenção combinada do HIV e registrar características de utilização dos serviços de saúde. Con- sideramos variáveis referentes ao acesso (se recebeu preservativos e gel lubrificante) e a utilização de insumos de prevenção ao HIV (preservativo feminino, masculino, creme/gel lubrificante, tratamento de HIV e sífilis, vacinação de hepatite B, PrEP e PEP) e características de utilização dos serviços de saúde (local que recebeu insumos de prevenção e/ou participou de palestras nos últimos seis meses; local que procurou na ocorrência de uma IST; se buscou assistência quando sofreu violência física, verbal ou sexual; e se ela se identifica como MTS nos serviços de saúde).

$\mathrm{Na}$ análise descritiva, foram calculadas as prevalências das variáveis de interesse, após o ajuste para os diversos portes populacionais das 12 cidades (em que o tamanho da amostra de cada uma havia sido fixado em 350), e a correção da homofilia, que consiste na tendência a recrutar pessoas com características semelhantes, requisito metodológico da técnica de amostragem RDS.

Foram investigados os fatores associados a dois desfechos de interesse "conhecimento sobre PEP e PrEP" e "uso de preservativo feminino". Inicialmente foram realizadas análises bivariadas e aplicados testes de hipóteses correspondentes (qui-quadrado e quando necessário teste de Fisher), calculados os respectivos intervalos de confiança de $95 \%$, as razões de prevalência e os respectivos valores $p$. Posteriormente foi utilizado o modelo de regressão de Poisson.

As variáveis "escolaridade", "identificação como trabalhadora do sexo no serviço de saúde", "local de Trabalho" e "tempo de exposição na profissão" foram dicotomizadas para facilitar a análise.

A ordem de entrada de cada uma das variáveis no modelo seguiu segundo o nível de significância e a força de associação. As variáveis que entraram no modelo deveriam ter nível de significância de p-valor $<0,10)$. Foram avaliados ajustes do modelo verificando se alguma variável perdia significância. Em caso positivo, esta era removida e entrava a próxima variável conforme sequência. Este foi o procedimento utilizado para estimar cada um dos modelos, sendo a medida de associação o oddsratio e seu intervalo de confiança de $95 \%$.

$\mathrm{O}$ projeto de pesquisa foi aprovado pelo Comitê de Ética em Pesquisa da Oswaldo Cruz Fundação. O banco de dados foi cedido pelo Departamento de Doenças Crônicas e Infecções Sexualmente Transmissíveis da Secretaria de Vigilância em Saúde do Ministério da Saúde DCCI/ SVS/MS ${ }^{19,20}$. 


\section{Resultados}

$\mathrm{Na}$ Tabela 1 podemos observar as principais características relacionadas ao acesso e a utilização de insumos de prevenção combinada. A maioria das participantes recebeu preservativos masculinos nos últimos seis meses $(82,0 \%)$, porém cerca de $66 \%$ e $60 \%$ declararam não ter recebido preservativo feminino e creme/gel lubrificante respectivamente, no mesmo período.

Cerca de 37\% das participantes não estava vacinada contra hepatite B e um percentual de $16,5 \%$ desconhece seu status vacinal. Dentre àquelas MTS que referem ter sido vacinadas, 14,6\% não lembra o número de doses.

No que diz respeito ao tratamento para HIV, entre aquelas mulheres que conhecem seu diagnóstico, um percentual importante de cerca de $29 \%$ interrompeu e ou não iniciou o tratamento.

Para o tratamento da sífilis foi encontrado um menor percentual de mulheres que não realizaram tratamento após o diagnóstico (12,2\%).

A profilaxia pós exposição (PEP) foi utilizada por apenas $7,1 \%$ das mulheres entrevistadas.

A Tabela 2 apresenta informações sobre os serviços de saúde e tipo de assistência recebida pelas MTS. Nesta tabela é possível observar que o serviço público de saúde foi o local em que a maioria teve acesso a insumos e informações sobre prevenção.

Em relação à ocorrência de infecções sexualmente transmissíveis (IST) noúltimo ano, o local preferencial para atendimento foi o posto de saúde ou unidade de saúde da família (51,3\%). Cerca de $17 \%$ referiu não ter procurado nenhum serviço.

Mais de 70\% das mulheres que sofreram violência física ou sexual não procuraram um serviço de saúde e pouco mais da metade das participantes do estudo referiu nunca se identificar como MTS nos serviços de saúde (51,5\%).

A Tabela 3 apresenta informações relacionadas aos fatores associados ao conhecimento de PEP e PrEP a partir do modelo logístico proposto. Nesta tabela observamos que MTS com maior escolaridade e aquelas que trabalham em locais fechados tem respectivamente $50 \%$ e $25 \%$ mais chance de ter conhecimento sobre a profilaxia pós exposição (PEP).

Em contrapartida, MTS que não participam ou são membros de ONG (RP: 0,60; IC: 95\%\%; $0,45-, 0,83)$ que não participaram de palestras ou receberam materiais sobre prevenção nos últimos seis meses (RP: 0,67; IC: 95\%: 0,56-0,83) que não procuram o mesmo serviço de saúde
Tabela 1. Descrição do acesso e utilização de métodos de prevenção combinada entre mulheres trabalhadoras do sexo, em doze cidades Brasileiras, 2016.

\begin{tabular}{|c|c|c|c|}
\hline Acesso & n & $\%$ & IC \\
\hline \multicolumn{4}{|l|}{ Preservativo masculino } \\
\hline Sim & 3.449 & 81,9 & $80,2-83,4$ \\
\hline Não & 764 & 18,1 & $16,6-19,8$ \\
\hline \multicolumn{4}{|l|}{ Preservativo feminino } \\
\hline $\operatorname{Sim}$ & 1.407 & 33,5 & $31,6-35,5$ \\
\hline Não & 2.790 & 66,5 & $64,5-68,4$ \\
\hline \multicolumn{4}{|l|}{ Creme/gel lubrificante } \\
\hline $\operatorname{Sim}$ & 1.697 & 40,3 & $38,4-42,3$ \\
\hline Não & 2.509 & 59,7 & $57,7-61,6$ \\
\hline Utilização & $\mathbf{n}$ & $\%$ & IC \\
\hline \multicolumn{4}{|l|}{ Preservativo feminino } \\
\hline Sim, frequentemente & 262 & 6,2 & $5,3-7,3$ \\
\hline Sim, uso eventual & 1.039 & 24,7 & $23,1-26,4$ \\
\hline Não, nunca uso & 2.900 & 69 & $67,2-70,8$ \\
\hline Vacina Hepatite B & $\mathbf{n}$ & $\%$ & IC \\
\hline Não & 1.568 & 37,4 & $35,6-39,3$ \\
\hline $\operatorname{Sim}(1,2$ ou 3 doses $)$ & 1.319 & 31,5 & $29,7-33,3$ \\
\hline $\begin{array}{l}\text { Sim, mas não lembra } \\
n^{\circ} \text { doses }\end{array}$ & 612 & 14,6 & $13,2-16,1$ \\
\hline Não sabe & 691 & 16,5 & $15,1-18,0$ \\
\hline Tratamento HIV & $\mathbf{n}$ & $\%$ & IC \\
\hline Sim, faço & 64 & 71,3 & $57,7-80,3$ \\
\hline Já fiz, mas interrompi & 4 & 4,1 & $1,5-10,8$ \\
\hline Não & 22 & 24,6 & $14,9-36,7$ \\
\hline Tratamento Sífilis & $\mathbf{n}$ & $\%$ & IC \\
\hline Sim & 423 & 87,8 & $83,8-90,9$ \\
\hline Não & 59 & 12,2 & $9,1-16,2$ \\
\hline PEP & $\mathbf{n}$ & $\%$ & IC \\
\hline Sim & 131 & 7,1 & $5,7-8,8$ \\
\hline Não & 1.729 & 92,9 & $91,2-94,3$ \\
\hline
\end{tabular}

IC: Intervalo de Confiança.

Fonte: Elaborado pelos autores.

(RP: 0,74; IC: 95\%: 0,60-0,92) e aquelas que não se identificam como trabalhadora do sexo no serviço de saúde (RP: 0,74; IC: 95\%: 0,61-0,91) estão no grupo de trabalhadoras que tem menos chances de ter conhecimento sobre a PEP. Em todas as análiseshouve significância estatística.

No que diz respeito à PrEP foi possível identificar associação entre menor conhecimento sobre a profilaxia entre as mulheres que não participam de ONG (RP: 0,49; IC: 95\%; 0,35-0,69) não receberam materiais sobre prevenção nos últimos seis meses (RP: 0,65; IC95\%; 0,50-0,85) e não se identificam como trabalhadoras do sexo nosserviços de saúde (RP: 0,56; IC: 95\%; 0,44-0,74). 
Tabela 2. Descrição de Serviços e Assistência à Saúde.

\begin{tabular}{|c|c|c|c|}
\hline Local que recebeu as camisinhas femininas de graça & $\mathbf{n}$ & $\%$ & IC \\
\hline Em um serviço público de saúde & 904 & 64,2 & $60,9-67,3$ \\
\hline Em uma associação ou ONG & 159 & 11,3 & $9,3-13,7$ \\
\hline No local que trabalho como trabalhadora do sexo & 271 & 19,2 & $16,8-22,0$ \\
\hline Em bares, boates, termas ou saunas & 11 & 0,8 & $0,4-1,4$ \\
\hline Ganhei de um (a) agente de prevenção & 204 & 14,5 & $12,2-17,1$ \\
\hline \multicolumn{4}{|l|}{ Local que participou da palestra e/ou recebeu o material educativo } \\
\hline Em um serviço público de saúde & 522 & 50,8 & $47,0-54,6$ \\
\hline Em uma associação ou ONG & 177 & 17,3 & $14,5-20,3$ \\
\hline No local que trabalho como trabalhadora do sexo & 174 & 17 & $14,5-19,7$ \\
\hline Em outro lugar & 176 & 17,2 & $14,5-20,4$ \\
\hline $\begin{array}{l}\text { Nos últimos } 12 \text { meses, você teve algum dos problemas relacionados às doenças } \\
\text { sexualmente transmissíveis? Qual serviço você procurou primeiramente? }\end{array}$ & $\mathbf{n}$ & $\%$ & IC \\
\hline Farmácia & 254 & 18,2 & $15,8-21,0$ \\
\hline Posto de saúde ou unidade de saúde da família & 714 & 51,3 & $47,8-54,6$ \\
\hline SAE, Centro de Especialidades, Policlínica pública ou PAM & 27 & 1,9 & $1,1-3,3$ \\
\hline UPA ou outra unidade de pronto atendimento público & 36 & 2,6 & $1,7-4,0$ \\
\hline Ambulatório de hospital público & 29 & 2,1 & $1,3-3,2$ \\
\hline Consultório particular & 80 & 5,8 & $4,4-7,5$ \\
\hline Pronto-atendimento ou emergência de hospital privado & 7 & 0,5 & $0,2-1,3$ \\
\hline Médico ou profissional de saúde do local que trabalho & 6 & 0,4 & $0,1-1,3$ \\
\hline Nenhum serviço & 240 & 17,2 & $14,0-19,9$ \\
\hline $\begin{array}{l}\text { Por causa de violência física ou verbal, você recebeu algum tipo de assistência de } \\
\text { saúde? }\end{array}$ & $\mathbf{n}$ & $\%$ & IC \\
\hline $\operatorname{Sim}$ & 187 & 22,6 & $19,2-26,5$ \\
\hline Não & 638 & 77,4 & $73,5-80,8$ \\
\hline Por causa de violência sexual, você procurou algum tipo de assistência de saúde? & $\mathrm{n}$ & $\%$ & IC \\
\hline Sim & 288 & 26,5 & $23,3-30,0$ \\
\hline Não & 797 & 73,5 & $70,0-76,7$ \\
\hline Quando você vai ao serviço de saúde, você diz que você é trabalhadora do sexo? & $\mathrm{n}$ & $\%$ & IC \\
\hline Sim, sempre & 1.014 & 24,3 & $22,7-25,9$ \\
\hline Só às vezes & 626 & 15 & $13,6-16,4$ \\
\hline Geralmente não & 386 & 9,2 & $8,1-10,5$ \\
\hline Nunca me declarei como trabalhadora do sexo & 2.153 & 51,5 & $49,6-53,5$ \\
\hline
\end{tabular}

Fonte: Elaborado pelos autores.

A Tabela 4 apresenta informações relacionadas aos fatores associados ao uso do preservativo feminino. Nesta tabela é possível observar que mulheres que trabalham em locais fechados (RP: 0,77; IC: 95\%0,61-0,99), não são membros de ONG (RP: 0,57; IC: 95\%; 0,40-0,83), não receberam material educativo sobre IST e/ou não participaram de palestras nos últimos seis meses (RP: 0,57; IC95\%; 0,57-0,94) e também não se identificam como trabalhadora do sexo nos serviços de saúde (RP: 0,54; IC: 95\%\%; 0,42-0,70) tendem a usar menos o preservativo feminino.

Mulheres que realizam trabalho sexual há mais de 5 anos (RP: 1,31; IC: 95\%\%; 1,02-1,70) e que referem usar preservativo durante sexo vaginal com parceiro fixo em mais da metade das vezes em que o encontro sexual ocorre (RP: 2,39; IC: $95 \% ; 1,57-3,65)$ tem mais chances de usar preservativo feminino.

\section{Discussão}

Apesar do percentual elevado de mulheres que recebem preservativos masculinos $(81,9 \%)$, cerca de dois terços das participantes referiram não ter recebido preservativo feminino. A maior chance de utilização do preservativo feminino está relaciona- 
Tabela 3. Análises bivariada e multivariada dos fatores associados ao conhecimento de PEP e PrEP entre mulheres trabalhadoras do sexo de doze cidades Brasileiras, 2016.

\begin{tabular}{|c|c|c|c|c|c|c|c|c|}
\hline \multirow{3}{*}{ Fatores } & \multicolumn{4}{|c|}{ Conhecimento PEP } & \multicolumn{4}{|c|}{ Conhecimento de PrEP } \\
\hline & \multicolumn{2}{|c|}{ Bivariada } & \multicolumn{2}{|c|}{ Multivariada } & \multicolumn{2}{|c|}{ Bivariada } & \multicolumn{2}{|c|}{ Multivariada } \\
\hline & $\mathbf{R P}$ & IC 95\% & RP & IC 95\% & RP & IC 95\% & RP & IC 95\% \\
\hline \multicolumn{9}{|l|}{ Escolaridade } \\
\hline $\begin{array}{l}\text { Elementar Incompleto até } \\
\text { Fundamental Incompleto }\end{array}$ & 1,000 & & 1,000 & & 1,000 & & - & - \\
\hline $\begin{array}{l}\text { Fundamental Completo ou } \\
\text { mais }\end{array}$ & 1,571 & $1,32-1,87$ & 1,492 & $1,25-1,79$ & 1,186 & $0,92-1,52$ & - & - \\
\hline \multicolumn{9}{|l|}{ Local de Trabalho } \\
\hline Rua & 1,000 & & 1,000 & & 1,000 & & - & - \\
\hline Local fechado & 1,457 & $1,21-1,75$ & 1,275 & $1,05-1,55$ & 1,041 & $0,80-1,35$ & - & - \\
\hline \multicolumn{9}{|l|}{ Membro de ONG } \\
\hline Sim & 1,000 & & 1,000 & & 1,000 & & 1,000 & \\
\hline Não & 0,506 & $0,38-0,67$ & 0,609 & $0,45-0,83$ & 0,426 & $0,30-0,59$ & 0,493 & $0,35-0,69$ \\
\hline \multicolumn{9}{|l|}{$\begin{array}{l}\text { Participou de palestra e/ou } \\
\text { recebeu material sobre IST nos } \\
\text { últimos seis meses }\end{array}$} \\
\hline $\operatorname{Sim}$ & 1,000 & & 1,000 & & 1,000 & & 1,000 & \\
\hline Não & 0,603 & $0,50-0,73$ & 0,679 & $0,56-0,83$ & 0,569 & $0,44-0,74$ & 0,654 & $0,50-0,85$ \\
\hline \multicolumn{9}{|l|}{$\begin{array}{l}\text { Costuma procurar o mesmo } \\
\text { serviço de saúde }\end{array}$} \\
\hline $\operatorname{Sim}$ & 1,000 & & 1,000 & & 1,000 & & - & - \\
\hline Não & 0,719 & $0,59-0,88$ & 0,742 & $0,60-0,92$ & 0,945 & $0,71-1,26$ & - & - \\
\hline \multicolumn{9}{|l|}{$\begin{array}{l}\text { Se identifica com trabalhadora } \\
\text { do sexo no serviço de saúde }\end{array}$} \\
\hline $\operatorname{Sim}^{\star}$ & 1,000 & & 1,000 & & 1,000 & & 1,000 & \\
\hline Não* & 0,739 & $0,61-0,90$ & 0,747 & $0,61-0,91$ & 0,537 & $0,41-0,69$ & 0,569 & $0,44-0,74$ \\
\hline
\end{tabular}

RP: razão de prevalência; IC: intervalo de confiança.

Fonte: Elaborado pelos autores.

da à realização de trabalho sexual por mais de cinco anos e uso de preservativo com parceiro fixo.

Dados divulgados pelo Ministério da Saúde sobre a Pesquisa de Conhecimentos Atitudes e Práticas na População Brasileira (PCAP), realizada em 2013, apontou que apesar do elevado nível de conhecimento das mulheres sobre o preservativo feminino ( $85 \%)$, sua utilização é baixa $(5,0 \%)$. A pesquisa também não apontou diferenças significativas no uso do preservativo relacionadas à escolaridade e à classe econômica ${ }^{11}$.

O preservativo feminino foi incorporado aos serviços de saúde desde o ano 2000, porém sua distribuição não acompanhou grandes campanhas de orientação sobre o seu uso. Houve pouca divulgação, comercialização e em alguns momentos interrupção de sua disponibilidade, fatores que mantiveram o seu uso muito limitado ${ }^{12}$.

O preservativo feminino de borracha nitrílica não é alergênico e pode ser colocado com até oito horas de antecedência, o que se torna uma facilidade em contextos de trabalho sexual, em que frequentemente é necessária uma negociação quanto ao uso do preservativo. Dessa forma, a mulher passa a ocupar um lugar central e decisivo relacionado ao grau de risco ao qual pretende se submeter ${ }^{12}$.

Grangeiro et al. ${ }^{21}$ estudaram os métodos de prevenção e as intervenções estruturais que podem ter impacto na incidência de HIV. Um dos resultados aponta para a importante contribuição do preservativo feminino na redução de riscos para MTS em situação de violência ou que fazem programas sem a utilização do preservativo pelo cliente buscando maior remuneração ${ }^{21}$.

Entre as intervenções biomédicas que compõem o escopo da prevenção combinada ao HIV está a vacinação contra hepatite B. Em nossa análise, cerca de $31 \%$ estariam elegíveis à vacinação, ou seja, deveriam estar vacinadas, mas não estão 
Tabela 4. Análises bivariada e multivariada dos fatores associados ao uso/utilização de preservativos femininos por mulheres trabalhadoras do sexo de doze cidades brasileiras. Brasil, 2016.

\begin{tabular}{|c|c|c|c|c|}
\hline \multirow{3}{*}{ Fatores } & \multicolumn{4}{|c|}{ Uso Preservativo Feminino } \\
\hline & \multicolumn{2}{|c|}{ Bivariada } & \multicolumn{2}{|c|}{ Multivariada } \\
\hline & RP & IC 95\% & RP & IC 95\% \\
\hline \multicolumn{5}{|l|}{ Local de trabalho } \\
\hline Rua & 1,000 & & 1 & \\
\hline Local fechado & 0,758 & $0,63-0,91$ & 0,7780462 & $0,61-0,99$ \\
\hline \multicolumn{5}{|l|}{ Tempo de exposição na profissão } \\
\hline$<5$ anos & 1,000 & & 1 & \\
\hline 5 ou mais & 1,346 & $1,11-1,62$ & 1,313703 & $1,02-1,70$ \\
\hline \multicolumn{5}{|l|}{ Participa ou é membro de ONG } \\
\hline Sim & 1,000 & & 1 & \\
\hline Não & 0,509 & $0,38-0,67$ & 0,5768557 & $0,40-0,83$ \\
\hline \multicolumn{5}{|c|}{$\begin{array}{l}\text { Nos últimos } 6 \text { meses você participou de palestra e/ou } \\
\text { recebeu material educativo sobre IST }\end{array}$} \\
\hline $\operatorname{Sim}$ & 1,000 & & 1,000 & \\
\hline Não & 0,713 & $0,59-0,86$ & 0,734 & $0,57-0,94$ \\
\hline \multicolumn{5}{|c|}{$\begin{array}{l}\text { Nos últimos } 6 \text { meses, com que frequência vocês usaram } \\
\text { camisinha no sexo vaginal com o(s) parceiro(s) fixo(s)? }\end{array}$} \\
\hline Nenhuma das vezes & 1,000 & & 1,000 & \\
\hline Menos da metade das vezes & 1,211 & $0,84-1,74$ & 1,356 & $0,93-1,98$ \\
\hline Mais da metade das vezes & 2,438 & $1,63-3,64$ & 2,396 & $1,57-3,65$ \\
\hline Todas as vezes & 1,777 & $1,38-2,28$ & 1,744 & $1,35-2,25$ \\
\hline \multicolumn{5}{|c|}{$\begin{array}{l}\text { Você se identifica como trabalhadora do sexo no serviço } \\
\text { de saúde }\end{array}$} \\
\hline $\operatorname{Sim}$ & 1,000 & & 1,000 & \\
\hline Não & 0,579 & $0,48-0,70$ & 0,541 & $0,42-0,70$ \\
\hline
\end{tabular}

RP: razão de prevalência; IC: intervalo de confiança.

Fonte: Elaborado pelos autores.

e/ou não receberam todas as doses para garantir a imunização.

O desconhecimento sobre o risco da hepatite B e falta de acesso aos serviços de saúde são algumas das situações que dificultam a imunização de populações de maior vulnerabilidade.

Quanto às trabalhadoras do sexo portadoras de HIV, encontramos cerca de $29 \%$ que relataram não realizar tratamento e/ou tê-lo interrompido. Atualmente existem evidências irrefutáveis (estudos HPTN $052^{22}$, Partner ${ }^{23}$ e Opposites Attract $\left.{ }^{24}\right)$, que demonstram que PVHIV em tratamento e com boa adesão à terapia antirretroviral (TARV), atingem rapidamente a supressão viral, representando um impacto extremamente positivo na redução da transmissão do HIV, embasando as atuais recomendações de tratamento como prevenção ${ }^{21-25}$.

Carvalho et al ${ }^{26}$ realizaram uma revisão integrativa de literatura e identificaram múltiplos fatores associados à adesão à TARV, estando en- tre eles questões individuais; características do tratamento; características da infecção, aspectos da relação com o serviço de saúde e apoio social. De acordo com essas variáveis, a adesão assumiu valores diferentes dependendo da população estudada, confirmando o caráter heterogêneo e regional dos fatores relacionados à adesão ${ }^{26}$.

Ampliar o número de PVHIV em tratamento e diminuir o número de abandono da TARV são dois desafios importantes para o Brasil e para os demais países da América Latina. Estima-se que, ao final de 2018, havia aproximadamente 900 mil PVHIV no Brasil, das quais 766 mil (85\%) estavam diagnosticadas. Destas, 594 mil (66\%) estavam em tratamento e $62 \%$ (554 mil) estavam com carga viral suprimida ${ }^{5,12}$. Na América Latina, em 2018, 80\% das PVHIV estavam diagnosticadas, entre essas $62 \%$ estavam em tratamento e $55 \%$ atingiram a supressão viral ${ }^{4}$.

No contexto do trabalho sexual, o enfrentamento desse desafio precisa considerar as especi- 
ficidades e vulnerabilidades que o acompanham como, por exemplo, as barreiras enfrentadas por essa população para chegar aos serviços de saúde, incluindo estigma e discriminação.

Internacionalmente alguns estudos vêm demonstrando vantagens do início de tratamento do HIV fora dos serviços de saúde desde que a TARV seja iniciada com segurança. Estudo realizado em Malawi identificou um maior número de pessoas que iniciam tratamento em casa do que àquelas que foram encaminhadas a um serviço de saúde. Para Ford et al., iniciar a TARV fora dos serviços de saúde pode ampliar e melhorar o acesso ao tratamento do HIV, principalmente para populações com dificuldades de acesso. $\mathrm{Na}$ Tanzânia essa possibilidade melhorou as taxas de início de TARV e reduziu o abandono de tratamento entre trabalhadores do $\mathrm{sexo}^{27}$.

A indicação de que a PEP foi pouco utilizada pelas MTS $(7,1 \%)$ aliado ao fato de que mulheres com maior escolaridade e que trabalham em locais fechados teriam mais chance de ter conhecimento sobre a PEP são informações que podem embasar a ampliação e a qualificação da oferta de PEP no país, visto que a maioria das trabalhadoras do sexo trabalha na rua e tem baixa escolaridade, de acordo com os achados de Szwarcwald et al., em estudo realizado com a mesma população ${ }^{28}$.

Fatores associados ao menor conhecimento sobre PEP e PrEP estão relacionados à dificuldade de acesso à informação, não vinculação a um serviço de saúde e revelação de sua atividade laboral. Cohen et al. descreve entre as lições aprendidas no programa de PEP da cidade de São Francisco (EUA), que a falta de conhecimento da PEP entre potenciais usuários contribui para sua subutilização ${ }^{29}$. De maneira geral, as trabalhadoras do sexo não têm conhecimento suficiente sobre PEP e PrEP a ponto de usufruir de seus benefícios.

Os serviços públicos, especialmente as Unidades de Saúde da Família, são os locais que as trabalhadoras do sexo procuram quando se trata de um problema relacionado às infecções sexualmente transmissíveis (IST) (51,3\%). Em contrapartida, àquelas que foram vítimas de violência física ou sexual não buscaram qualquer tipo de assistência. Essa é uma informação importante que pode apoiar a organização das Redes de Atenção às Vítimas de Violência a partir da articulação dos serviços de atenção primária com demais equipamentos de saúde e proteção social. A ampliação desse tipo de serviço deve necessariamente estar ligada à oferta das tecnologias de prevenção combinada.
Outro achado importante foi relacionado à necessidade de vinculação a uma ONG para que as MTS tenham mais acesso à informação. Além disso, essa articulação entre ONG e setores públicos de saúde e de proteção social poderia potencializar os efeitos positivos da prevenção do HIV relacionados ao empoderamento das MTS.

Percentual importante das participantes deste estudo omite ser trabalhadora do sexo quando busca um serviço de saúde (51,5\%). Dourado et al. apontam o estigma e a discriminação como as principais barreiras de acesso aos serviços de saúde. Os autores referem que a adoção da invisibilidade é uma estratégia para evitar atitudes discriminatórias por parte dos profissionais de saúde ${ }^{6}$.

\section{Limitações}

Além das limitações próprias da metodologia RDS descritas por Damacena et al. ${ }^{20}$, podemos citar o fato de que o instrumento de coleta de informações durante a entrevista não contemplava questões relacionadas à prevenção combinada, modelo de prevenção adotado pelo Brasil. Outras limitações estão relacionadas às questões de utilização da PEP e conhecimento da PrEP pelas trabalhadoras do sexo, que poderiam ter considerado os momentos de disponibilização dessas tecnologias nos serviços de saúde. Para utilização da PEP, a indicação clínica pode ter se constituído como um viés importante de utilização.

\section{Conclusão}

Mesmo com as limitações desse estudo, foi possível identificar que as trabalhadoras do sexo ainda não têm acesso assegurado às tecnologias de prevenção combinada no Brasil, especialmente à utilização de preservativo feminino e PEP, realização de tratamento para HIV e vacinação para hepatite B. Fica evidente, neste estudo, que as barreiras de acesso, principalmente aquelas relacionadas ao estigma e à discriminação impedem que as MTS possam se beneficiar de tais tecnologias, inclusive em situações que envolvem violência.

O perfil de MTS que tem menor conhecimento sobre as intervenções biomédicas (PEP e PrEP) foi associado a não vinculação a uma $\mathrm{ONG}$, não participação em palestras e/ou acesso a informações sobre prevenção nos últimos seis meses. Também foi associado com o fato de não se identificarem como MTS nos serviços de saúde. 
Dessa forma, consideramos que a ampliação da oferta de prevenção combinada em Serviços de Atenção Primária (APS), a adoção de modelos de cuidados diferenciados e a promoção de um ambiente livre de discriminação podem contribuir de maneira expressiva para que essas tecnologias alcancem quem mais iria se beneficiar delas, a exemplo das MTS, evitando novas infecções.

\section{Colaboradores}

Os autores contribuíram significativamente para o artigo e concordam com o conteúdo do manuscrito. AF Kolling e EM Hamann participaram da concepção e delineamento do estudo, análise e interpretação dos dados, redação do artigo e aprovação da versão final. SB Oliveira participou da análise estatística, interpretação dos dados, revisão do manuscrito e aprovação da versão final. 


\section{Referências}

1. Brasil. Ministério da Saúde (MS). Secretaría de Vigilancia em Saúde, Departamento de DST, Aids e Hepatites Virais (BR). Boletim Epidemiológico Aids e DST. Ano IV n 0. Brasília: MS;2017. [acessado 2020 mar 15]. Disponível em: http://www.aids.gov.br/pt-br/ pub/2017/boletim-epidemiologico-hivaids-2017

2. Brasil. Ministerio da Saúde (MS). Secretaria de Vigilância em Saúde, Departamento de DST AIDS e Hepatites Virais. Relatório de Monitoramento Clínico do HIV. 2017; 94. Brasília: MS;2017. [acessado 2020 mar 15]. Disponível em: http://www.aids.gov.br/pt -br/pub/2017/relatorio-de-monitoramento-clinicodo-hiv

3. Drew M, Guimarães C, Ferreira-j C. Prevalence estimates of HIV, syphilis, hepatitis $B$ and $C$ among female sex workers (FSW) in Brazil, 2016. 2018; 97(Supl. 1):38.

4. AVERT. HIV and AIDS in Latin America regional overview. 2015 [Internet] [cited 2020 Mar 15]. Available from: http://www.avert.org/professionals/hiv-around -world/latin-america/overview

5. Crabtree-ram B, Belaunzar PF, Sued O, Sierra-madero J, Cahn P, Pozniak A, Grinsztejn B. The HIV epidemic in Latin America: a time to reflect on the history of success and the challenges ahead. 2020;1-4.

6. Dourado I, Guimarães MDC, Damacena GN, Magno L, De Souza Júnior PRB, Szwarcwald CL, Brazilian FSW Group. Sex work stigma and non-disclosure to health care providers: Data from a large RDS study among FSW in Brazil. BMC Int Health Hum Rights 2019; 19(1):1-8

7. Chacham AS, Diniz SG, Maia MB, Galati AF, Mirim LA. Sexual and Reproductive Health Needs of Sex Workers: Two Feminist Projects in Brazil. Reprod Health Matters 2007; 15(29):108-118.

8. Baral S, Beyrer C, Muessig K, Poteat T, Wirtz AL, Decker, MR Sherman SG, Kerrigan D. Burden of HIV among female sex workers in low-income and middle -income countries: A systematic review and meta-analysis. Lancet Infect Dis 2012; 12(7):538-549.

9. Dias LB. Uma reflexão crítica entre Prostituição e Políticas Públicas no Brasil. REP [Internet]. 2018; 2(1):4446. [acessado 2020 mar 4]. Disponível em: https://periodicos.unb.br/index.php/rep/article/view/7001

10. Paes F, Queiroz C, Primo SM. Os sistemas políticojurídicos da prostituição e a regulamentação como legitimadora da prática exploratória. Fibra Lex 2016; 1:1-19.

11. Brasil. Ministério da Saúde (MS). Secretaria de Vigilância em Saúde. Departamento de DST, Aids e Hepatites Virais. Pesquisa de Conhecimento, atitudes e práticas na população Brasileira. Série G. Estatística e Informação em Saúde. Brasília: MS; 2013. 166p.:il.

12. Fundo de População das Nações Unidas (UNFPA). Preservativo feminino: das políticas globais à realidade brasileira. 2011. [acessado 2020 mar 4]. Brasília: UNFPA; 1987. Disponível em: www.unfpa.org.br.

13. Brasil. Ministério da Saúde (MS). Secretaria de Vi- gilância em Saúde. Departamento de Vigilância, Prevenção e Controle das Infecções Sexualmente Transmissíveis, do HIV/Aids e das Hepatites Virais. Prevenção Combinada do HIV/Bases conceituais para profissionais, trabalhadores(as) e gestores(as) de saúde. Brasília: MS; 2017. 123 p.: il.11.

14. Brasil. Ministério da Saúde (MS). Secretaria de Vigilância em Saúde. Departamento de Vigilância, Prevenção e Controle das Infecções Sexualmente Transmissíveis, do HIV/Aids e das Hepatites Virais. Prevenção Combinada do HIV/Bases conceituais para profissionais, trabalhadores(as) e gestores(as) de saúde. Brasília: MS; 2017. 123 p.: il.11.

15. Brasil. Ministério da Saúde (MS). Secretaria de Vigilância em Saúde. Departamento de Doenças de Condições Crônicas e Infecções Sexualmente Transmissíveis (DCCI). Protocolo Clínico e Diretrizes Terapêuticas para Profilaxia Pós Exposição (PEP) de Risco à Infecção pelo HIV, IST e Hepatites Virais; Brasília:MS; 2018. [acessado 2020 mar 4]. Disponível em: http://www.aids.gov.br/pt-br/pub/2015/protocoloclinico-e-diretrizes-terapeuticas-para-profilaxia-pos -exposicao-pep-de-risco

16. Brasil. Ministério da Saúde (MS). Secretaria de Vigilância em Saúde. Departamento de Doenças de Condições Crônicas e Infecções Sexualmente Transmissíveis (DCCI). Relatório de monitoramento clínico do HIV. Brasília:MS; 2019. [acessado 2020 mar 4]. Disponível em: http://www.aids.gov.br/pt-br/pub/2019/ relatorio-de-monitoramento-clinico-do-hiv-2019

17. Brasil. Ministério da Saúde (MS). Secretaria de Vigilância em Saúde. Departamento de Doenças de Condições Crônicas e Infecções Sexualmente Transmissíveis (DCCI). Relatório de Implantação da Profilaxia Pós Exposição PrEP HIV 2019; Brasília:MS; 2019. [acessado 2020 mar 4]. Disponível em: http://www. aids.gov.br/pt-br/pub/2019/relatorio-de-implantacao-da-profilaxia-pos-exposicao-prep-hiv

18. Brasil. Ministério da Saúde (MS). Profilaxia pré-exposição (PrEP) de risco à infecção pelo HIV [Internet]. Vol. 1, Secretaria de Vigilância em Saúde. Departamento de Vigilância, Prevenção e Controle das Infecções Sexualmente Transmissíveis, do HIV/Aids e das Hepatites Virais. Protocolo Clínico e Diretrizes Terapêuticas. 2018. 47 p. [acessado 2020 mar 4]. Disponível em: http://www.aids.gov.br/publicacao/2017/ protocolo-clinico-e-diretrizes-terapeuticas-para-profilaxia-pre-exposicao-prep-de-ri

19. Szwarcwald CL, Damacena GN, Souza-Júnior P, Guimarães M, Almeida W, Souza Ferreira AP, FerreiraJúnior O, Dourado I, Brazilian FSW Group. Brazilian FSW Group (2018). Factors associated with HIV infection among female sex workers in Brazil. Medicine 2018; 97(Supl. 1):S54-S61.

20. Damacena GN, Szwarcwald CL, Souza Júnior PRB, Ferreira Júnior OC, Almeida WS, Pascom ARP, Pimenta MC. Application of the Respondent-Driven Sampling methodology in a biological and behavioral surveillance survey among female sex workers, Brazil, 2016. Revista Brasileira de Epidemiologia 2019; 22(Supl. 1)::e190002. 
21. Grangeiro A, Ferraz D, Calazans G, Zucchi EM, Díaz-Bermúdez XP, Grangeiro A, et al. The effect of prevention methods on reducing sexual risk for HIV and their potential mpact on a large-scale: a literature review. Rev Bras Epidemiol 2015; 18(Supl. 1):43-62.

22. Cohen MS, Chen YQ, McCauley M, Gamble T, Hosseinipour MC, Kumarasamy N, Hakim JG, Kumwenda J, Grinsztejn B, Pilotto JH, Godbole SV, Mehendale S, Chariyalertsak S, Santos BR, Mayer KH, Hoffman IF, Eshleman SH, Piwowar-Manning E, Wang L, Makhema J, Mills LA, de Bruyn G, Sanne I, Eron J, Gallant J, Havlir D, Swindells S, Ribaudo H, Elharrar V, Burns D, Taha TE, Nielsen-Saines K, Celentano D, Essex M, Fleming TR, HPTN 052 Study Team. Antiretroviral therapy for the prevention of HIV-1 transmission. New Engl J Med 2016; 375(9):830-839.

23. Vernazza P, Collins S, van Lunzen J, Corbelli GM, Estrada V, Geretti AM, Beloukas A, Asboe D, Viciana P, Gutiérrez F, Clotet B, Pradier C, Gerstoft J, Weber R, Westling K, Wandeler G, Prins JM, Rieger A, Stoeckle M, Kümmerle T, Bini T, Ammassari A, Gilson R, Krznaric I, Ristola M, Zangerle R, Handberg P, Antela A, Allan S, Phillips AN, Lundgren J, PARTNER Study Group. Sexual Activity Without Condoms and Risk of HIV Transmission in Serodifferent Couples When the HIV-Positive Partner Is Using Suppressive Antiretroviral Therapy. JAMA 2016; 316(2):171-181.

24. Bavinton BR, Prestage GP, Jin F, Phanuphak N, Grinsztejn B, Fairley CK, Baker D, Hoy J, Templeton DJ, Tee BK, Kelleher A, Grulich AE, Opposites Attract Study Group. Strategies used by gay male HIV serodiscordant couples to reduce the risk of HIV transmission from anal intercourse in three countries. J Int AIDS Society 2019; 22(4): e25277.

25. Brasil. Ministério da Saúde (MS). Manejo da infecção pelo hiv em adultos [Internet]. Secretaria de Vigilância em Saúde. Departamento de Vigilância, Prevenção e Controle das Infecções Sexualmente Transmissíveis, do HIV/Aids e das Hepatites Virais. Protocolo. 2018. 412 p. [acessado 2020 mar 4]. Disponível em: http:// www.aids.gov.br/pt-br/pub/2013/protocolo-clinico -e-diretrizes-terapeuticas-para-manejo-da-infeccao -pelo-hiv-em-adultos.
26. Carvalho PP, Barroso SM, Coelho HC, Penaforte FRO. Factors associated with antiretroviral therapy adherence in adults: An integrative review of literature. Cien Saude Colet 2019; 24(7):2543-2555.

27. Ford N, Geng E, Ellman T, Orrell C, Ehrenkranz P, Sikazwe I, Jahn A, Rabkin M, Ayisi Addo S, Grimsrud A, Rosen, S, Zulu I, Reidy W, Lejone T, Apollo T, Holmes C, Kolling AF, Phate Lesihla R, Nguyen HH, Bakashaba B, Bygrave $\mathrm{H}$. Emerging priorities for HIV service delivery. PLoS Med 2020;17(2): e1003028.

28. Szwarcwald CL, Almeida S, Damacena GN. Changes in attitudes, risky practices, and HIV and syphilis prevalence among female sex workers in Brazil from 2009 to 2016. Med 2018; 97(Supl. 1):S46-S53.

29. Cohen SE, Liu AY, Bernstein KT, Philip S. Preparing for HIV pre-exposure prophylaxis: Lessons learned from post-exposure prophylaxis. Am J Prev Med 2013; 44(1 Supl. 2):S80-S85.

Artigo apresentado em 26/03/2020

Aprovado em 08/06/2020

Versão final apresentada em 10/06/2020

Editores-chefes: Romeu Gomes, Antônio Augusto Moura da Silva 